\section{Integración del internet de las cosas en la gestión de la cadena de suministro de alimentos: una revisión bibliométrica}

\author{
Alcibíades Rodríguez (D), Karen Ochoa (D, \\ Práxedes Torres \\ Facultad de Ingeniería Industrial, Universidad Tecnológica de Panamá. \\ alcibiades71@gmail.com; choakaren0517@gmail.com; \\ praxedes.torres@utp.ac.pa \\ DOI: 10.33412/pri.v12.1.2448
}

\section{(2)(1) $\$($}

Resumen: Se estima que el internet de las cosas (IoT por sus siglas en inglés) tendrá un rol sustancial en el desarrollo de nuevos paradigmas de gestión de cadena de suministros. La integración de las tecnologías de loT en las diversas fases de la cadena de suministro contribuirá a solventar los retos de control, monitoreo, y planeación que mantienen las cadenas de suministros tradicionales. En el caso específico de la gestión de cadenas de suministro de alimentos, existen actualmente desafíos importantes derivados de la complejidad en cuanto al manejo y control de los productos perecederos. Sin embargo, a pesar del potencial que brinda el loT, su integración en la gestión de cadenas de suministro es un tema que aún se encuentra en sus primeras etapas de desarrollo. Razón por la cual, la presente investigación explora el impacto que tiene la integración del loT en la gestión de la cadena de suministro de alimentos, a través de una revisión sistemática de la literatura.

Palabras claves: Cadena de suministro de alimentos, logística de alimentos, internet de las cosas.

Title: Integration of the internet of things in the food supply chain management: a systematic review of the literature.

\begin{abstract}
It is estimated that the internet of things (IoT) will play a substantial role in the development of new supply chain management paradigms. The integration of loT technologies in the various phases of the supply chain will help solve the control, monitoring, and planning challenges that traditional supply chains maintain. In the specific case of food supply chain management, there are currently significant challenges arising from the complexity of handling and controlling perishable products. However, despite the potential offered by loT, its integration into supply chain management is an issue that is still in its early stages of development. For this reason, this research explores the impact
\end{abstract}

that the integration of loT has on the management of the food supply chain, through a systematic review of the literature.

Key words: food supply chain, food logistics, internet of things.

Tipo de artículo: Investigación.

Fecha de recepción: 3 de agosto de 2020.

Fecha de aceptación: 5 de enero de 2021.

\section{Introducción}

Hoy en día se vive probablemente el momento más cercano a lo que representó para el hombre, el monumental avance tecnológico-industrial registrado durante la primera revolución industrial. Este conjunto de fenómenos sociales, industriales y tecnológicos ha pasado a denominarse: la cuarta revolución industrial o en términos más contemporáneos, la Industria 4.0

Muchas serán las tecnologías que acompañarán este proceso, sin embargo, [1] perfilan 9 de ellas como las más relevantes, estas son: big data, robots autónomos, simulación, integración horizontal y vertical, la nube, fabricación aditiva, realidad aumentada, seguridad cibernética e internet de las cosas (IoT). La loT ha cobrado especial relevancia para el desarrollo de este trabajo, ya que la integración de este concepto con la cadena de suministro da paso a una serie de aplicaciones que sin duda revolucionarán la manera como se gestionan los procesos industriales.

El loT corresponde a una red de objetos físicos los cuales se encuentran interconectados con la finalidad de brindar información a la empresa en tiempo real, permitiendo mayor agilidad, visibilidad y mejorando las actividades de rastreo, de manera que se facilitan los procesos de planeación, control y coordinación [2].

Hoy en día, las sofisticadas estructuras que conforman las cadenas de suministro, integradas en un sistema que se encuentra en constante cambio, las hace vulnerables a un sin número de riesgos en todos sus niveles [3]. En este sentido el loT ofrece la capacidad de llegar a niveles de control, planificación y optimización remota en tiempo real [4].

La cadena de suministro de alimentos (Food Supply Chain FSC) según estadísticas de la FAO, presenta una grave problemática en cuanto al desperdicio de alimentos a nivel mundial. Esto resulta alarmante y pone de manifiesto una problemática que trasciende el aspecto meramente productivo, para retratar una realidad social que resulta inaceptable tomando en cuenta la gran cantidad de personas que padecen hambre alrededor del mundo. Actualmente las cadenas de suministro de alimentos representan una compleja estructura de eslabones que van desde las actividades productivas primarias 0 agrícolas, pasando por los distribuidores, y llegando al consumidor final a través de actividades de procesamiento, logística y control de los productos alimentarios.

Los alimentos están en riesgo de sufrir múltiples daños a lo largo de la cadena [5], por lo que se requiere que los actores involucrados en su gestión sean capaces de controlar y supervisar 
las condiciones de los productos, así como de garantizar la calidad e inocuidad a lo largo de todo el proceso [6].

En función de comprender las implicaciones que podría tener la integración del loT en las diversas fases de la cadena de suministro de alimentos, se ha planteado identificar a través de una revisión de literatura, los principales aportes científicos que se han realizado en los últimos años referentes a este tema. De esta forma se podrán obtener datos cuantificables, que permitan plantear un panorama general acerca de la literatura existente con lo que se contribuya a visibilizar posibles rutas de solución a la problemática en la gestión de la cadena de suministro de alimentos mediante el uso del loT.

\section{Concepto del internet de las cosas (IoT)}

Múltiples definiciones de loT han sido propuestas a pesar de que este concepto es bastante reciente. Según [7], sintácticamente, este término está compuesto por dos palabras, en donde el término "internet" está orientado a la red y el segundo "cosas" enfocado a los objetos genéricos para integrarlos en un marco común. En este sentido han sido propuestas varias definiciones, a continuación, se detallan algunas de ellas:

Punto de interacción entre los humanos y las aplicaciones mediante la nueva dimensión de la comunicación y la integración de las cosas [8].

Red global integrada por numerosos dispositivos conectados que dependen de tecnologías sensoriales, comunicaciones y redes de procesamiento de información [9].

Se conforma de elementos digitales y físicos que se pueden vincular por medio de tecnologías de información que permiten nuevas clases de aplicaciones y servicios [10].

Los planteamientos anteriores llevan a concluir que el loT es una red global de objetos conectados a internet y entre ellos, de manera que pueden intercambiar y enviar información en tiempo real, acerca de un proceso específico, facilitando con esto la toma de decisiones.

Por otra parte, según [11] las tecnologías de loT deben contar con 4 características esenciales que son: interconectividad entre dispositivos, heterogeneidad para que puedan interactuar entre diferentes plataformas, ser resistentes a cambios dinámicos y su comunicación debe ser a gran escala.

\subsection{El loT en la gestión de la cadena de suministro de alimentos}

La gestión de la cadena de suministro podría definirse como un proceso que involucra: la planificación de las actividades referentes al abastecimiento, transformación, almacenaje y distribución de alimentos, de manera tal que se logre una optimización total de las actividades involucradas, conllevando con esto el aumento del valor agregado de los alimentos que llegan al consumidor final y al costo mínimo para la empresa.

Hoy en día se requiere atender a millones de personas alrededor del mundo que precisan de alimentos frescos en sus mesas [12]. No obstante, al tratarse de suministros perecederos se tiene un reto extra en cuanto al cumplimiento de las funciones logísticas básicas, de llevar: los productos correctos, en las cantidades y calidad adecuada, en el tiempo correcto, al lugar correcto y por el precio correcto [13].

Por lo que, ante estos desafíos, la industria de alimentos perecederos deberá adaptar sus modelos de cadena de suministro, con ayuda de sistemas vinculados al loT, de manera que puedan lidiar con situaciones impredecibles a la vez que cumplen con los requisitos de calidad para alimentos de manera integral [14].

\section{Metodología}

Este trabajo de investigación se dirige a través de una búsqueda bibliográfica exhaustiva en cuatro (4) bases de datos científicas (Emerald, Science Direct, Wiley Online Library y Springer), a conocer el estado del arte del tema objeto de estudio.

En total fueron consultados 245 artículos preliminares, correspondientes al periodo incluido entre 2010 y 2019. Posteriormente los artículos preliminares fueron depurados para dar con un total final de 135 artículos científicos.

El trabajo investigativo constó de dos etapas: la primera corresponde a una revisión de literatura, que sirvió para encontrar los artículos relevantes del tema de investigación. Durante la segunda etapa se organizó toda la información encontrada de manera cuantitativa, haciendo uso de un análisis bibliométrico.

Para la realización de búsqueda de información se utilizó "La metodología para la realización de búsquedas sistemáticas de literatura" propuesta por [15] que consta de cinco (5) etapas que son: identificación del campo de estudio, selección de fuentes de información, realización de la búsqueda, gestión y depuración de resultados y análisis de los resultados.

Las publicaciones encontradas fueron clasificadas atendiendo al aporte que realizan las mismas a cada eslabón específico de la cadena de suministro de alimentos. Para dicha clasificación se utilizó el criterio presentado por [16] el cual segmenta la cadena de suministro de alimentos en 8 etapas: producción, procesamiento, embalaje, ventas y marketing, logistica y distribución, retail, consumidor y residuos.

Además de las 8 categorías propuestas se consideró pertinente agregar las categorías de trazabilidad que cuenta con un número plural de estudios, y la de enfoque múltiple para estudios que abarquen aplicaciones del loT en más de un eslabón de la cadena de suministro de alimentos. De esta manera se conforman las 10 categorías finales, bajo las cuales se clasificaron cada uno de los 135 estudios identificados en la revisión sistemática de literatura.

\section{Resultados}

Por medio de la aplicación sistemática de la metodología propuesta por [15] se lograron identificar 135 artículos científicos que esbozan el estado del arte del tema objeto de estudio. En el presente apartado se evalúan estos resultados por medio de un análisis bibliométrico.

La figura 1 muestra la distribución cronológica de las publicaciones identificadas. Resulta evidente que ha habido un incremento notorio en el volumen de publicaciones a partir del año 2013. Esta tendencia ha representado que sólo el periodo 
comprendido entre 2016-2019 cuente con el $85 \%$ de las publicaciones identificadas, siendo esto un indicador claro del interés investigativo creciente en este campo. Por otra parte, la tabla 1 muestra las revistas científicas con mayores aportes a este trabajo, siendo las más destacadas: Computers and Electronics in Agriculture e Industrial Management \& Data Systems con 9 y 6 aportes respectivamente. Lo anterior demuestra un interés marcado de estas dos revistas en temas referentes a posibles aplicaciones de sistemas tecnológicos en la solución de problemáticas agrícolas e industriales.

De igual forma se realizó una categorización atendiendo al tipo de metodología utilizada en cada artículo identificado (figura 2), siendo la implementación de modelos y prototipos la de mayor preferencia investigativa con un $57.78 \%$ de los estudios, seguida por el planteamiento de diseños conceptuales con un $13.33 \%$ y los estudios de revisión con un $11.85 \%$. Estos resultados indican un enfoque centrado mayormente en la resolución de los problemas que mantiene la cadena de suministro de alimentos a través de prototipos robóticos, sistemas de comunicación, softwares, etc.

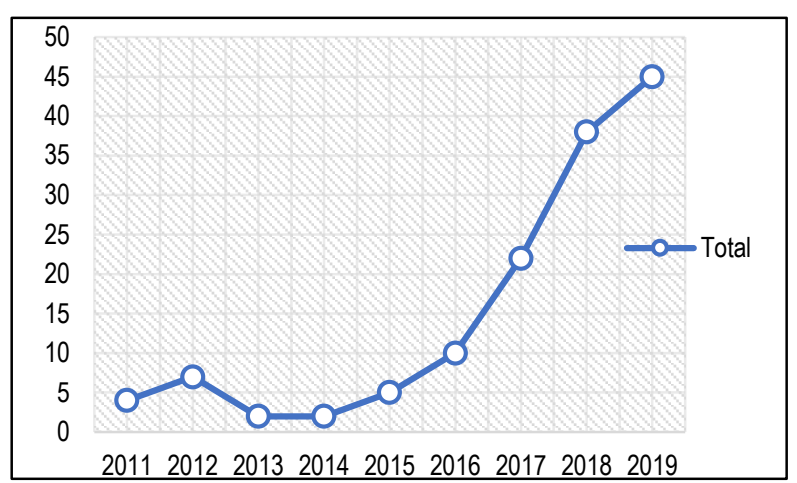

Figura 1. Distribución cronológica de las publicaciones.

Tabla 1. 10 principales revistas con contenido relevante para esta investigación.

\begin{tabular}{|l|c|c|}
\hline \multicolumn{1}{|c|}{ Revistas } & Cantidad & SJR \\
\hline $\begin{array}{l}\text { Computers and } \\
\text { Electronics in } \\
\text { Agriculture }\end{array}$ & 9 & 0.95 \\
\hline $\begin{array}{l}\text { Industrial Management } \\
\text { \& Data Systems }\end{array}$ & 6 & 1.14 \\
\hline $\begin{array}{l}\text { Procedia Computer } \\
\text { Science }\end{array}$ & 5 & 0.28 \\
\hline Food Control & 3 & 1.45 \\
\hline $\begin{array}{l}\text { Sustainable } \\
\text { Computing: Informatics } \\
\text { and Systems }\end{array}$ & 4 & 0.26 \\
\hline $\begin{array}{l}\text { Biosystems } \\
\text { Engineering }\end{array}$ & 2 & 0.83 \\
\hline $\begin{array}{l}\text { Concurrency and } \\
\text { Computation: Practice } \\
\text { and Experience }\end{array}$ & 2 & 0.31 \\
\hline $\begin{array}{l}\text { Neural Computing and } \\
\text { Applications }\end{array}$ & 2 & 0.64 \\
\hline Waste Management & 2 & 1.52 \\
\hline $\begin{array}{l}\text { Wireless Personal } \\
\text { Communications }\end{array}$ & 2 & 0.25 \\
\hline
\end{tabular}

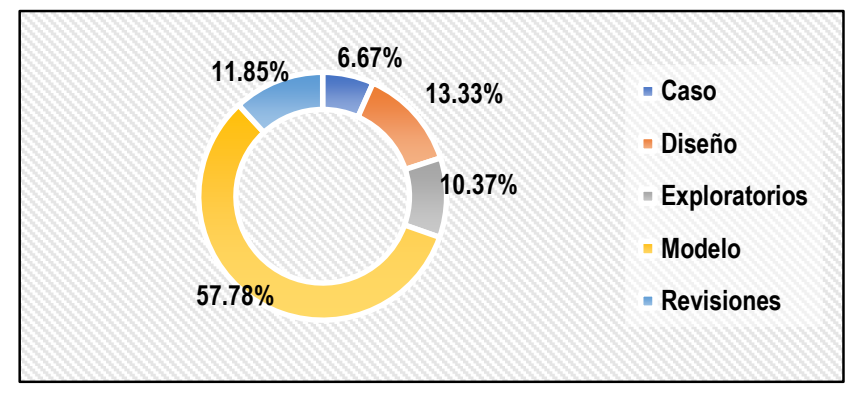

Figura 2. Distribución porcentual de metodologías.

En cuanto a la distribución regional de las publicaciones se observa una marcada tendencia en el volumen investigativo de las regiones asiáticas (tabla 2), las cuales han aportado la mayor parte $(66 \%)$ de las investigaciones que fueron identificadas en este estudio.

Tabla 2. 10 principales países con mayor productividad respecto al tema en estudio.

\begin{tabular}{|c|c|c|c|}
\hline País & Cantidad & País & Cantidad \\
\hline China & 50 & Brasil & 3 \\
\hline India & 32 & Ecuador & 3 \\
\hline Estados Unidos & 5 & Inglaterra & 2 \\
\hline
\end{tabular}

Particularmente China con 50 publicaciones se coloca como el país de mayores aportes respecto a la temática investigada. Estos resultados son consistentes con otras investigaciones que fueron consultadas durante la realización de este trabajo, y en donde igualmente las instituciones chinas figuran como las primeras en volumen investigativo.

\subsection{Distribución porcentual de las categorías desarrolladas en las publicaciones identificadas.}

Tal como se observa (figura 3), cuatro categorías (producción, procesos, trazabilidad y enfoque múltiple) representan el $91.86 \%$ de los estudios identificados, mientras que las restantes 6 categorías (consumidor, embalaje, logística, residuos, retail y ventas) representan el restante $8.14 \%$.

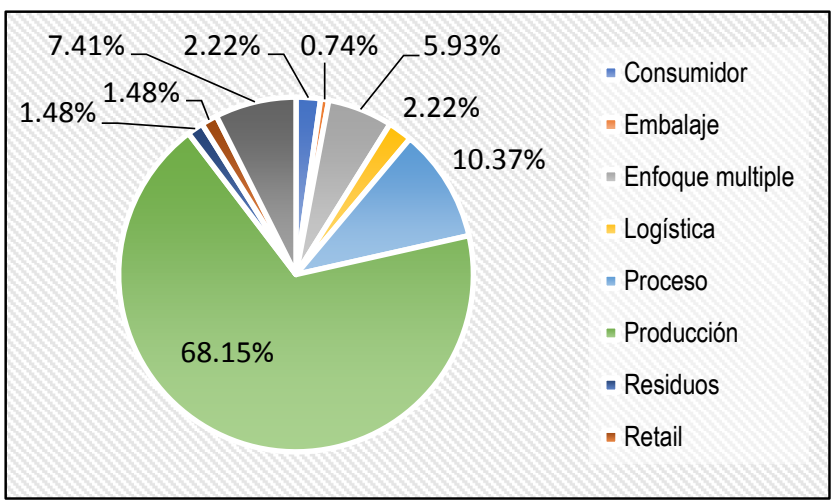

Figura 3. Distribución porcentual de categorías temáticas. 
Es importante aclarar que la categoría "ventas" no se encuentra representada dentro de la gráfica anterior ya que ninguno de los estudios identificados mantenía contenido referente a este ámbito. Según lo consultado, la mayor parte de los investigadores mantienen un enfoque hacia el desarrollo de proyectos que integran tecnologías de loT en el eslabón de "producción" de la cadena de suministro de alimentos, es decir en la etapa relacionada con las actividades productivas primarias 0 agrícolas.

Así pues, la mayor parte de las líneas investigativas se han enfocado en los aspectos relacionados al mejoramiento en la gestión de las actividades agrícolas tradicionales a través del uso de las diferentes herramientas tecnológicas de gestión que ofrece el internet de las cosas.

\subsection{Inventario final de categorías y subcategorías temáticas}

A continuación, se presenta un inventario final (tabla 3 ) de las categorías y subcategorías temáticas finales, así como de la cantidad de artículos identificados en cada una de ellas.

Tabla 3. Resumen de categorías y subcategorías temáticas finales.

\begin{tabular}{|c|c|c|}
\hline Categoría & Subcategoría & Total \\
\hline \multirow{11}{*}{ Producción } & Agricultura inteligente & 21 \\
\hline & Acuaponía & 1 \\
\hline & Agricultura & 28 \\
\hline & $\begin{array}{l}\text { Agricultura de } \\
\text { precisión }\end{array}$ & 11 \\
\hline & $\begin{array}{l}\text { Cadena de suministro } \\
\text { agrícola }\end{array}$ & 3 \\
\hline & Cultivos & 10 \\
\hline & Hidroponía & 2 \\
\hline & Invernadero & 4 \\
\hline & Irrigación & 8 \\
\hline & Maquinaria & 3 \\
\hline & Suelo & 1 \\
\hline \multirow{3}{*}{ Procesos } & Cadena de frío & 2 \\
\hline & Calidad alimentaria & 7 \\
\hline & Seguridad alimentaria & 5 \\
\hline Embalaje & - & 1 \\
\hline \multirow{2}{*}{ Logística } & Almacenamiento & 1 \\
\hline & Logística alimentaria & 2 \\
\hline Retail & - & 2 \\
\hline Consumidor & - & 3 \\
\hline Residuos & - & 2 \\
\hline Trazabilidad & - & 10 \\
\hline Enfoque múltiple & - & 8 \\
\hline \multicolumn{2}{|c|}{ Total } & 135 \\
\hline
\end{tabular}

4.3 Análisis de las aplicaciones del internet de las cosas en cada eslabón de la cadena de suministro de alimentos.

Se hizo un análisis del rol que juega el loT en el mejoramiento de cada etapa de la FSC en base a las publicaciones científicas identificadas durante la revisión sistemática de literatura.

Producción: una de las áreas más prometedoras en este eslabón es la de agricultura inteligente, dentro de la cual se distinguen numerosas aplicaciones tecnológicas en los ámbitos de fertilización, monitoreo, micro irrigación [17-37] así como en el de acuaponía [38]. Igualmente se observa un interés científico creciente en el ámbito agrícola en general y en la solución de las problemáticas tradicionales de este sector, específicamente existe un énfasis en aplicaciones como control de pestes, gestión de las enfermedades de los cultivos, gestión inteligente del ganado etc. [39-66]. En el campo de la agricultura de precisión se observa una prevalencia de investigaciones enfocadas en el uso del internet de las cosas para recolección de datos que faciliten la toma de decisiones oportuna [67-77]. El ámbito de gestión integral de cadena de suministro agrícola y sus implicaciones ha sido abordado de igual forma por algunos investigadores [78-80]. En cuanto al uso del internet de las cosas en la gestión específica de ciertos cultivos se pueden identificar estudios tendientes a optimizar la producción, monitorear el crecimiento, así como a generar alertas tempranas contra plagas [81-90]. Por otra parte, se encuentran artículos sobre el uso del loT en la optimización de métodos controlados de cultivo como la hidroponía [91, 92] y los invernaderos [93-96]. En esta misma línea las preocupaciones sobre la disponibilidad del agua han planteado la posibilidad de buscar en el internet de las cosas un medio que contribuya la administración eficiente de este recurso [97-104]. Por último, se encuentran estudios referentes a conocer las implicaciones del uso de loT en ámbitos como la gestión de maquinaria agrícola [105-107] y la administración del suelo [108].

Proceso: uno de los factores más preocupantes durante los últimos años en materia de calidad alimentaria son las condiciones de vulnerabilidad que mantienen los alimentos a lo largo de la cadena de suministros. Se identificaron estudios en las áreas de cadena de frío [109, 110], calidad alimentaria [111-117] y seguridad alimentaria [118-122].

Embalaje: en el ámbito de embalaje se destaca el estudio referente al empacado inteligente a través de loT en las cadenas de suministro alimentarias de Brasil [123].

Logística: la preservación de la calidad alimentaria en el proceso de transporte ha sido objeto de análisis por parte de algunos estudios. Se ha hecho énfasis de manera específica en la habilitación de camiones refrigerados con herramientas de loT que contribuyan a optimizar esta actividad [124]. De igual manera se enfatiza en el uso de ambientes de loT para la creación de rutas de distribución inteligentes [125]. En cuanto a las labores logísticas de almacenamiento se identificó la investigación sobre el uso de internet de las cosas para el monitoreo del almacenaje de granos [126].

Retail / Minorista: en este eslabón de la cadena de suministro de alimentos se puede destacar el estudio sobre las barreras 
existentes en la implementación de un modelo de loT en las actividades de retail [127], así como la investigación sobre la generación de valor en tiendas de abarrotería por medio del loT [128].

Consumidor: las tecnologías de loT han tenido un impacto que trasciende el aspecto industrial, y llega a tener aplicaciones incluso a nivel doméstico. $Y$ es que con el nivel de vida tan ocupado que llevan las personas hoy en día, es difícil gestionar tareas tan comunes como la administración de la despensa. En este sentido el internet de las cosas ofrece soluciones que permiten la posibilidad de conocer el inventario que mantenemos de ciertos alimentos en casa $[129,130]$. En esta misma línea se detectaron estudios que brindan aplicaciones para facilitar el pedido a domicilio de alimentos [131].

Residuos: la gestión residuos es una de las actividades de las cuales menor volumen de investigación científica se ha identificado, sin embargo, se observan algunas investigaciones en las áreas de reducción de desperdicios [132] y gestión de residuos en restaurantes [133].

Trazabilidad: durante los últimos años se ha generado el concepto "Farm to table (del campo a la mesa)" para hacer referencia entre otras cosas al hecho de que el consumidor final pueda tener certeza acerca de la procedencia de sus alimentos. En este contexto muchos estudios han tratado de garantizar esta premisa haciendo uso del loT y técnicas de trazabilidad. En el área agrícola destacan numerosas investigaciones [134- 138]. Así mismo otras líneas investigativas han optado por el estudio en concreto de la trazabilidad de productos específicos como es el caso del: pepino [139], patos [140,141] y comida pre empacada [142]. Los sistemas autónomos de trazabilidad [143] también se perfilan como un nuevo paradigma en la industria alimentaria.

Enfoque múltiple: en la categoría de enfoque múltiple se encontraron estudios que examinan el impacto integral del loT a lo largo de la FSC. En este sentido se observan estudios enfocados en el incremento de las ganancias [144, 145] la generación de modelos conceptuales [146], la virtualización de la cadena de suministro de alimentos [147] y el mejoramiento de la eficiencia y control $[148,149]$. Así mismo se pudieron identificar dos revisiones de literatura $[150,151]$ que plantean aplicaciones del loT en diferentes ámbitos de la FSC.

\section{Discusión}

Existe un interés creciente en el desarrollo de investigaciones concernientes al impacto que puede tener el uso del internet de las cosas en los diversos eslabones de la cadena de suministro de alimentos. Este aspecto es corroborado con los resultados cronológicos que fueron planteados en apartados previos y en donde se observa un desarrollo investigativo creciente a partir del año 2013. Existe una tendencia investigativa marcada hacia el desarrollo de estudios en la categoría de producción. Esta área en especial de la FSC se presenta como una de las más prometedoras si se toma en cuenta la amplia cantidad de modelos aplicativos y de prototipos que fueron identificados.

El loT optimizará la FSC ofreciendo entre otras cosas mejores sistemas para la toma de decisiones, capacidades de monitoreo en tiempo real con alertas tempranas, sistemas de utilización racional de insumos, lo cual ofrecerá beneficios notables en los costos de producción, automatización de procesos y maximización de los márgenes de beneficio.

Por otra parte, los estudios de [152, 148] validan que la mayor parte de las investigaciones se han restringido a eslabones específicos de la cadena de suministro. El trabajo de [43] concuerda en que la mayor parte del volumen investigativo respecto al tópico en estudio se ha desarrollado principalmente en países asiáticos, siendo China el de mayor desarrollo. Mientras que la investigación de [42] respalda el potencial especial que mantiene el loT en la optimización del eslabón de producción de la FSC.

\section{Conclusión}

El internet de las cosas ofrece beneficios fundamentalmente en la optimización de todos los eslabones de la FSC. No obstante, se encontró que la mayoría de las investigaciones se encuentran confinadas en ciertos eslabones de la FSC (producción, proceso, trazabilidad) lo cual ha contribuido a que no exista un desarrollo completo del tema en estudio. Se pudo determinar que el interés generalizado en el tema comienza a partir del año 2013, mayoritariamente en la forma de modelos aplicativos y con una prevalencia de investigaciones centradas en la solución individualizada de las problemáticas más que en una visión holística de los beneficios del loT en la FSC. Se lograron identificar vacíos en la literatura en cuanto a: beneficios económicos potenciales, costos de implementación, así como de las competencias requeridas para una correcta integración del loT en las actividades de la FSC. Es importante mencionar que los resultados que fueron obtenidos a través de la realización de este estudio complementan la investigación realizada por [152] respecto al rol potencial del loT en la gestión de la cadena de suministro. En este sentido se brinda una visión mucho más amplia del impacto del uso de las tecnologías de loT en el caso específico de la FSC, con lo cual se constituye una hoja de ruta para el desarrollo de futuras investigaciones.

\section{Referencias}

[1] M. Rüßmann, M. Lorenz, P. Gerbert y W. Manuela, «Industry 4.0: The Future of Productivity and Growth in Manufacturing Industries,»Boston Consulting Group, pp. 1-14, 2015.

[2] K. Butner, «The smarter supply chain of the future,» Strategy \& Leadership, pp. 22-31, 2010.

[3] M. Christopher y M. Holweg, «Supply Chain 2.0: managing supply chains in the era of turbulence,» International Journal of Physical Distribution \& Logistics Management: Vol 41, pp. 63-82, 2011

[4] FAO, «Food Wastage Footprint,» 2013.

[5] CCA, «Caracterización y gestión de la pérdida y el desperdicio de alimentos en América del Norte.,» Informe sintético, Comisión para la Cooperación Ambiental, 2017.

[6] R. Montanari, «Cold chain tracking: a managerial perspective,» Trends in Food Science \& Technology, pp. 425-431, 2008.

[7] R. Jedermann, L. Ruiz-Garcia y W. Lang, «Spatial temperature profiling by semi-passive RFID loggers for perishable food transportation,» Computers and Electronics in Agriculture, pp. 145-154, 2009.

[8] H. Sundmaeker, P. Guillemin, P. Friess y S. Woelfflé, «Vision and Challenges for Realising the Internet of Things,» European Union, 2010. 
[9] T. Lu y W. Neng, «Future internet: The Internet of Things,» de 2010 3rd International Conference on Advanced Computer Theory and Engineering(ICACTE), Chengdu, 2010.

[10] D. Miorandi, S. Sicari, F. De Pellegrini y I. Chlamtac, «Internet of things: Vision, applications and research challenges," Ad Hoc Networks, pp. 14979-1516, 2012.

[11] L. Atzori, A. lera y G. Morabito, "The Internet of Things: A survey,» Computer Networks: Vol. 54, pp. 2787-2805, 2010.

[12] International Telecomunication Union, «Descripción general de Internet de los objetos,» ITU, Ginebra, 2012.

[13] Y. Zhang, L. Zhao y C. Qian, «Modeling of an loT-enabled supply chain for perishable food with two-echelon supply hubs,» Industrial Management \& Data Systems, Vol. 117, pp. 1890-1905, 2017.

[14] C. Eksoz, A. Mansouri y M. Bourlakis, "Collaborative forecasting in the food supply chain: A conceptual framcework,» Int. J. Production Economics, Vol. 158, pp. 120-135, 2014.

[15] C. Medina-Lopez, J. A. Marin-Garcia y R. Alfalla-Luque, «Una propuesta metodológica para la realización de búsquedas sistemáticas de bibliografía,» WPOM-Working Papers on Operations Management, Vol. 1 , p. 13, 2010

[16] L. Ramundo, M. Taisch y S. Terzi, «State of the art of technology in the food sector value chain towards the IOT,» 2016 IEEE 2nd International Forum on Research and Technologies for Society and Industry Leveraging a Better Tomorrow, pp. 1-6, 2016.

[17] A. Sinha, G. Shrivastava y P. Kumar, «Architecting user-centric internet of things for smart agriculture,» Sustainable Computing: Informatics and Systems, 2019.

[18] K. Archana P. y S. Shefali P., «loT based Smart.Farming : Feature subset selection for optimized high-dimensional data using improved GA based approach for ELM,» Computers and Electronics in Agriculture, 2019.

[19] M. A. Zamora-Izquierdo, J. Santa, J. A. Martínez, V. Martínez y A. F. Skarmeta, "Smart farming loT platform based on edge andcloud computing,» Biosystem Engineering, 2018.

[20] G. Lavanya, C. Rani y P. Ganeshkumar, «An automated low cost loT based Fertilizer Intimation System for smart agriculture,» Sustainable Computing: Informatics and Systems, 2019.

[21] M. Taneja, N. Jalodia, J. Byabazaire, A. Davy y C. Olariu, «SmartHerd management: A microservices-based fog computing-assisted loT platform towards data-driven smart dairy farming,» Software: Practice and Experience, 2019.

[22] R. Krushna Das, M. Panda y S. Shree Dash, «Smart Agriculture System in India Using Internet of Things,» de Soft Computing in Data Analytics, 2018.

[23] L. Kamlesh, G. Hemant, A. Niket y G. Shashank, «Development of loT for Smart Agriculture a Review,» de Advances in Intelligent Systems and Computing vol. 841, Singapore , 2019, pp. 425-432.

[24] Ö. Köksal y B. Tekinerdogan, «Architecture design approach for loT-based farm management information systems,» Precision Agriculture, 2018.

[25] J. Li, W. Gu y H. Yuan, «Research on IOT Technology Applied to Intelligent Agriculture,» de Proceedings of the 5th International Conference on Electrical Engineering and Automatic Control, 2016.

[26] S. Bhowmick, B. Biswas, M. Biswas, A. Dey, S. Roy y K. Subir, «Application of loT-Enabled Smart Agriculture in Vertical Farming,» de Advances in Communication, Devices and Networking, 2019.

[27] S. AlZu'bi, B. Hawashin, M. Mujahed, Y. Jararweh y B. B. Gupta, «An efficient employment of internet of multimedia things in smart and future agriculture,» Multimedia Tools and Applications, 2019.

[28] E. Symeonaki, K. Arvanitis y D. Piromalis, "Cloud Computing for IoT Applications in Climate-Smart Agriculture: A Review on the Trends and Challenges Toward Sustainability.,") de Innovative Approaches and Applications for Sustainable Rural Development., Cham, 2019.

[29] S. Abouzahir, M. Sadik y E. Sabir, «loT-Empowered Smart Agriculture: A Real-Time Light-Weight Embedded Segmentation System.,» de
Ubiquitous Networking. Lecture Notes in Computer Science, vol 10542. Cham, 2017.

[30] G. Balakrishna y R. M. Nageshwara, "Study Report on Using IoT Agriculture Farm Monitoring.," de Innovations in Computer Science and Engineering. Lecture Notes in Networks and Systems, vol 74, Singapore, 2019.

[31] J. Mahalakshmi, K. Kuppusamy, C. Kaleeswari y P. Maheswari, «loT Sensor-Based Smart Agricultural System,» de Emerging Technologies for Agriculture and Environment, 2019.

[32] S. Koshy, V. Srikanth, P. Rajgarhia, K. Chinnusamy, D. Prasad y S. Chunduri, «Application of the internet of things (IOT) for Smart.Farming: a case study on groundnut and castor pest and disease forewarning,» CSI Transactions on ICT, 2018.

[33] K. Kadam, G. Chavan, U. Chavan, R. Shah y P. Kumar, «Smart and Precision Polyhouse Farming Using Visible Light Communication and Internet of Things," de Intelligent Computing and Information and Communication. Advances in Intelligent Systems and Computing, vol 673., Singapore, 2018.

[34] C. Man, L. Guo, Y. Gao y Y. Zhang, « Wisdom Farm Internet of Things Software Design and Selection Program,» de Communications, Signal Processing, and Systems. , 2020.

[35] J. Zhang, P. Liu, W. Xue y Z. Rui, «Farmland Intelligent Information Collection System Based on NB-loT.,» de Cloud Computing and Security. ICCCS 2018. , 2018.

[36] H. Agrawal, J. Prieto, C. Ramos y J. M. Corchado, «Smart feeding in farming through loT in silos,» de Intelligent Systems Technologies and Applications 2016, 2016.

[37] H. Cadavid, W. Garzón, A. Pérez, G. López, C. Mendivelso y C. Ramírez, «Towards a Smart Farming Platform: From loT-Based Crop Sensing to Data Analytics.,» de Advances in Computing. CCC 2018., 2018.

[38] M. Odema, I. Adly, A. Wahba y H. Ragai, «Smart Aquaponics System for Industrial Internet of Things (IloT),» de Proceedings of the International Conference on Advanced Intelligent Systems and Informatics 2017, 2017.

[39] B. Yan, C. Yan, C. Ke y X. Tan, «Information Sharing in Supply Chain of Agricultural Products Based on the Internet of Things,» Industrial Management \& Data Systems, pp. 116 (7): 1397-1416, 2016.

[40] B. Yan, X.-h. Wu, B. Ye y Y.-w. Zhang, "Three-level supply chain coordination of fresh agricultural products in the Internet of Things,》 Industrial Management \& Data Systems, 2017.

[41] P. Jayashankar, S. Nilakanta, W. Johnston, P. Gill y R. Burres, «loT adoption in agriculture: the role of trust, perceived value and risk,» Journal of Business \& Industrial Marketing, 2018.

[42] A. Tzounis, N. Katsoulas, T. Bartzanas y C. Kittas, «Internet of Things in agriculture, recent advances and future challenges,» Biosystems Engineering, 2017.

[43] J. M. Talavera, L. E. Tobón, J. A. Gómez, M. A. Culman, J. M. Aranda, D. T. Parra, L. A. Quiroz, A. Hoyos y L. E. Garreta, «Review of loT applications in agro-industrial and environmental fields,» Computers and Electronics in Agriculture, 2017.

[44] J. L. Dos Santos, G. Pessin, C. Da Costa y R. Da Rosa, «AgriPrediction: A proactive internet of things model to anticipate problems and improve production in agricultural crops,» Computers and Electronics in Agriculture, 2019.

[45] H. Tse-Chuan, Y. Hongji, C. Yeh-Ching y H. Ching-Hsien, «A Creative loT agriculture platform for cloud fog computing,» Sustainable Computing: Informatics and Systems, 2018.

[46] L. Zhao, S. Yin, L. Liu, Z. Zhang y S. Wei, «A Crop Monitoring System Based on Wireless Sensor Network,» Procedia Environmental Sciences, 2011.

[47] L. Zhang, I. Dabipi y W. Brown Jr., «Internet of Things Applications for Agriculture,» de Internet of Things A to Z, 2018.

[48] F. Zhang, «Research on Applications of Internet of Things in Agriculture,» de Informatics and Management Science VI, 2013. 
[49] M. Gomes, «The Internet of Things as an Integrated Service Platform to Increase Value to the Agriculture Stakeholders," de Putting Tradition into Practice: Heritage, Place and Design, 2017.

[50] C. Panda y R. Bhatnagar, «Social Internet of Things in Agriculture: An Overview and Future Scope,» de Toward Social Internet of Things (SIoT): Enabling Technologies, Architectures and Applications, 2020, pp. 317-334.

[51] Q. Razi y V. Nath, «Design of Smart Embedded System for Agricultural Update Using Internet of Things,» de Nanoelectronics, Circuits and Communication Systems., 2019.

[52] X. Min y W. Kuang, «Study on the Ecological Farming Control System Based on the Internet of Things,» Wireless Personal Communications, vol. 102, p. 2955-2967, 2018

[53] F. Ferrández-Pastor, J. García-Chamizo, M. Nieto Hidalgo y J. MoraMartínez, «User-Centered Design of Agriculture Automation Systems Using Internet of Things Paradigm,» de Ubiquitous Computing and Ambient Intelligence., 2017.

[54] R. Gómez, K. Real, C. Morán, P. Grijalva y T. Recalde, «loT Applications in Agriculture: A Systematic Literature Review,» de ICT for Agriculture and Environment, 2019.

[55] L. Zhou, L. Song, C. Xie y J. Zhang, «Applications of Internet of Things in the Facility Agriculture.," de Computer and Computing Technologies in Agriculture VI, 2013.

[56] J. Yu y W. Zhang, «Study on Agricultural Condition Monitoring and Diagnosing of Integrated Platform Based on the Internet of Things.,》 de Computer and Computing Technologies in Agriculture VI. , 2013.

[57] F. Bamigboye y E. Ademola, «Internet of Things: The Present Status, Future Impacts and Challenges in Nigerian Agriculture,» de Internet of Things. Information Processing in an Increasingly Connected World, 2019.

[58] T. Tegegne, H. Balcha y M. Beyene, «Internet of Things Technology for Agriculture in Ethiopia: A Review,» de Information and Communication Technology for Development for Africa., 2019.

[59] H. Zhou, B. Liu y P. Dong, «The Technology System Framework of the Internet of Things and Its Application Research in Agriculture,» de Computer and Computing Technologies in Agriculture V, Beijing, 2011.

[60] Z. Hu, Y. Gao, M. Li, H. Li, X. Yang y Z. Ma, «An Agricultural Habitat Information Acquisition and Remote Intelligent Decision System Based on the Internet of Things,》 de Computer and Computing Technologies in Agriculture XI, 2019.

[61] S. Juhi Reshma y A. Pillai, «Impact of Machine Learning and Internet of Things in Agriculture: State of the Art.,» de Proceedings of the Eighth International Conference on Soft Computing and Pattern Recognition, 2018.

[62] H. Kang, J. Lee, B. Hyochan y S. Kang, «A Design of loT Based Agricultural Zone Management System.,» de Information Technology Convergence, Secure and Trust Computing, and Data Management., 2012.

[63] P. Jayaraman, D. Palmer, A. Zaslavsky, A. Salehi y D. Georgakopoulos, «Addressing Information Processing Needs of Digital Agriculture with OpenloT Platform,» de Interoperability and Open-Source Solutions for the Internet of Things., 2015.

[64] H. Wu, F. Chen, H. Hu, Q. Liu y S. Ji, «A Secure System Framework for an Agricultural IOT Application,» de Advances in Computer Science and Ubiquitous Computing, 2017.

[65] G. Suila, P. Czekalski, E. L. Molua y K. Grochla, «An Architectural Framework Proposal for loT Driven Agriculture,» Computer Networks , 2019.

[66] A. Merizig, H. Saouli, M. Zouai y O. Kazar, «An Intelligent Approach for Enhancing the Agricultural Production in Arid Areas Using IoT Technology," de Advanced Intelligent Systems for Sustainable Development , 2019.

[67] M. C. Vuran, A. Salam, R. Wong y S. Irmak, «Internet of underground things in precision agriculture: Architecture and technology aspects,» $\mathrm{Ad}$ Hoc Networks, 2018
[68] B. Mazon-Olivo, D. Hernández-Rojas, J. Maza-Salinas y A. Pan, «Rules engine and complex event processor in the context of internet of things for precision agriculture,» Computers and Electronics in Agriculture, pp. 347360, 2018.

[69] T. Popović, L. Nedeljko, P. Ana, Z. Žarko, K. Božo y D. Slobodan, «Architecting an loT-enabled platform for precision agriculture and ecological monitoring: A case study," Computers and Electronics in Agriculture, vol. 140, pp. 255-265, 2017.

[70] K. Abhishek y K. Sanmeet, «Evolution of Internet of Things (IOT) and its significant impact in the field of Precision Agriculture,» Computers and Electronics in Agriculture, vol. 157, pp. 218-231, 2019.

[71] K. Foughali, K. Fathalah y f. Ali, «Monitoring system using web of things in precision agriculture,» Procedia Computer Science, 2017.

[72] K. Foughali, K. Fathalah y f. Ali, «Using Cloud IOT for disease prevention in precision agriculture,» Procedia Computer Science, pp. 575-582, 2018.

[73] J. Gómez, A. Fernandez y M. Zúñiga, «Monitoring of Small Crops for the Measurement of Environmental Factors Through the Internet of Things (IOT),» de Technology Trends, 2018.

[74] R. Marcelino, L. Casagrande, R. Cunha, Y. Crotti y V. Gruber, «Internet of Things Applied to Precision Agriculture,» de Online Engineering \& Internet of Things. Lecture Notes in Networks and Systems, 2017.

[75] C. Dewi y R. Chen, «Decision Making Based on loT Data Collection for Precision Agriculture,» de Intelligent Information and Database Systems: Recent Developments, 2019.

[76] X. Feng, F. Yan y X. Liu, «Study of Wireless Communication Technologies on Internet of Things for Precision Agriculture,» Wireless Personal Communications, 2019.

[77] H. Agrawal, R. Dhall, K. S. S. Iyer y V. Chetlapalli, «An improved energy efficient system for loT enabled precision agriculture,» Journal of Ambient Intelligence and Humanized Computing, 2019.

[78] S. Luthra, S. K. Mangla, D. Garg y A. Kumar, «Internet of Things (IOT) in Agriculture Supply Chain Management: A Developing Country Perspective,» Emerging Markets from a Multidisciplinary Perspective. Advances in Theory and Practice of Emerging Markets., pp. 209-220, 2018.

[79] G. Zhang, «Research on the Optimization of Agricultural Supply Chain Based on Internet of Things,» de Computer and Computing Technologies in Agriculture VII, 2014.

[80] K. Leng, L. Jin, W. Shi y I. Van Nieuwenhuyse, «Research on agricultural products supply chain inspection system based on internet of things,» Cluster Computing, 2018.

[81] A. N. Harun, N. Mohamed, R. Ahmad, A. R. A. Rahim y N. N. Ani, «Improved Internet of Things (IOT) monitoring system for growth optimization,» Computers and Electronics in Agriculture, 2019.

[82] S. Trilles, J. Torres, O. Belmonte, J. Zarazaga, A. González y J. Huerta, «Development of an open sensorized platform in a smart agriculture context: A vineyard support system for monitoring mildew disease,» Sustainable Computing: Informatics and Systems, 2019.

[83] Y. Shiroma, H. Afuso, R. Suwa, A. Kinjo, Y. Tonooka, T. Kaga, I. Nagayama, S. Tamaki y G. Maharjan, «Development of higher yield and high-quality mango production system based on Internet of Things,» Electronics and Communications in Japan, 2019.

[84] M. A. M. R. Guillén, B. López y J. M. Cecilia, «A high-performance loT solution to reduce frost damages in stone fruits," Concurrency and Computation: Practice and Experience, 2019.

[85] E. Hincapié, J. Sánchez y J. A. Carbonell, «loT Network Applied to Agriculture: Monitoring Stations for Irrigation Management in Soils Cultivated with Sugarcane,» de Advances in Information and Communication Technologies for Adapting Agriculture to Climate Change II, 2018.

[86] D. Wang, T. Chen y J. Dong, «Research of the Early Warning Analysis of Crop Diseases and Insect Pests," de Computer and Computing Technologies in Agriculture VII, 2013. 
[87] R. Kumar, S. Kumar y R. Krishna, «Crop Monitoring Using loT: A Neural Network Approach,» de Soft Computing: Theories and Applications, 2018.

[88] P. Jawade, D. Chaugule, D. Patil y H. Shinde, «Disease Prediction of Mango Crop Using Machine Learning and loT,» de Advances in Decision Sciences, Image Processing, Security and Computer Vision , 2019.

[89] S. Donzia, H. Yeo y H. Kim, «Design and Evaluation of Soil pH loT Sensor Attribute for Rice Agriculture in Central Africa.,» de Applied Computing and Information Technology, 2019.

[90] J. Guillermo, A. García-Cedeño, D. Rivas-Lalaleo, M. Huerta y R. Clotet, «loT Architecture Based on Wireless Sensor Network Applied to Agricultural Monitoring: A Case of Study of Cacao Crops in Ecuador,» de Advances in Information and Communication Technologies for Adapting Agriculture to Climate Change II, 2018.

[91] M. Manav, S. Sameer, S. Suresh, J. Rijo y V. M., «loT based hydroponics system using Deep Neural Networks,》 Computers and Electronics in Agriculture, pp. 473-486, 2018.

[92] G. Marques, D. Aleixo y R. Pitarma, «Enhanced Hydroponic Agriculture Environmental Monitoring: An Internet of Things Approach,» de Computational Science - ICCS 2019, 2019.

[93] Y. Tang, M. Jia, Y. Mei, Y. Yu, J. Zhang, R. Tang y K. Song, «3D intelligent supplement light illumination using hybrid sunlight and LED for greenhouse plants,» Optik, 2019.

[94] J. Ruan, X. Hu, X. Hou, Y. Shi, F. T. S. Chan, X. Wang, M. Gunasekaran, G. Mastorakis, M. Constandinos X. y X. Zhao, «An loT-based E-business model of intelligent vegetable greenhouses and its key operations management issues,» Neural Computing and Applications, 2019.

[95] S. S. Rek, C. B. Kannamma y C. S. Sangamitra, «A Novel Approach of IoTBased Smart Greenhouse Farming System,» de Green Buildings and Sustainable Engineering, 2018.

[96] Q. Li y H. Wu, «Research on Vegetable Growth Monitoring Platform Based on Facility Agricultural IoT.,» de Communications in Computer and Information Science, Singapore, 2017

[97] S. Zhang, M. Wang, W. Shi y W. Zheng, «Construction of intelligent water saving irrigation control system based on water balance,» IFACPapersOnLine, 2018

[98] M. Hadipour, D. J. Farrokhi y S. M. Aghazadeh, «An experimental setup of multi-intelligent control system (MICS) of water management using the Internet of Thins (IoT),» ISA Transactions, 2019.

[99] N. K. Nawandar y V. R. Satpute, «loT based low cost and intelligent module for smart irrigation system,» Computers and Electronics in Agriculture, 2019.

[100] K. Lakshmiprabha y C. Govindaraju, «Hydroponic-based smart irrigation system using Internet of Things,» International Journal of Communication Systems, 2019.

[101] S. Koduru, V. Padala y P. Padala, «Smart Irrigation System Using Cloud and Internet of Things.,» de Proceedings of 2nd International Conference on Communication, Computing and Networking. , 2019.

[102] R. Muley y V. N. Bhonge, «Internet of Things for Irrigation Monitoring and Controlling,» de Computing, Communication and Signal Processing, 2018.

[103] R. Raut, H. Varma, C. Mulla y V. Pawar, «Soil Monitoring, Fertigation, and Irrigation System Using loT for Agricultural Application,» de Intelligent Communication and Computational Technologies, 2017.

[104] B. Keswani, A. G. Mohapatra, A. Mohanty, A. Khanna, J. J. P. C. Rodrigues, D. Gupta y V. H. C. De Albuquerque, «Adapting weather conditions based IoT enabled smart irrigation technique in precision agriculture mechanisms,» Neural Computing and Applications, 2018.

[105] R. Zhang, F. Hao y X. Suna, «The Design of Agricultural Machinery Service Management System Based on Internet of Thing,» Procedia Computer Science, pp. 53-57, 2017.

[106] X. Jin, K. Chen, J. Ji, K. D. Zhao y H. Ma, «Intelligent vibration detection and control system of agricultural machinery engine,» Measurement, 2019.

[107] Q. Zhou, J. Jiang, Z. Zhao, J. Zhong, B. Pan, X. Jin y Y. Sun, «Research on the Internet of Things Platform Design for Agricultural Machinery
Operation and Operation Management,» de Computer and Computing Technologies in Agriculture XI, 2019.

[108] Q. Wu, Y. Liang, Y. Li, X. Wang, L. Yang y X. Wang, «Factors acquisition and content estimation of farmland soil organic carbon based upon Internet of Things,» Chinese Geographical Science, 2017.

[109] Y. Tsang, K. Choy, C. Wu, G. Ho, H. Lam y P. Koo, «An Internet of Things (loT)-based risk monitoring system for managing cold supply chain risks,» Industrial Management \& Data Systems, 2018

[110] H. Luo, M. Zhu, S. Ye, H. Hou, Y. Chen y L. Bulysheva, «An intelligent tracking system based on internet of things for the cold chain,» Internet Research, 2016.

[111] Y. Liu, W. Han, Y. Zhang, L. Li, J. Wang y L. Zheng, «An Internet-of-Things solution for food safety and quality control: A pilot project in China,» Journal of Industrial Information Integration, pp. 1-7, 2016.

[112] J. Tervonen, «Experiment of the quality control of vegetable storage based on the Internet-of-Things,» Procedia Computer Science, 2018.

[113] Y. Tsang, K. Choy, C. Wu, G. Ho, H. Lam y V. Tang, «An intelligent model for assuring food quality in managing a multi-temperature,» Food Control, 2018.

[114] M. TahaJilani, M. Zaka Ur Rehman, K. Abid Muhmmad, C. Omer, A. A. Muhammad y T. Muhammad, «An implementation of loT-based microwave sensing system for the evaluation of tissues moisture,» Microelectronics Journal, 2019

[115] Y. Ya, C. Huihui, T. Ou, W. Zhou y T. Fan, «Cross perishable effect on optimal inventory preservation control,» European Journal of Operational Research, vol. 276, pp. 998-1012, 2019.

[116] R. Junhu y S. Yan, «Monitoring and assessing fruit freshness in IOT-based e-commerce delivery using scenario analysis and interval number approaches,» Information Sciences, vol. 373, pp. 557-570, 2016.

[117] K. Gupta y N. Rakesh, «loT-Based Solution for Food Adulteration,» de Proceedings of First International Conference on Smart System, Innovations and Computing, 2018.

[118] K. Harpreet, «Modelling internet of things driven sustainable food security system,» Benchmarking: An International Journal, 2019.

[119] J. Wang y H. Yue, «Food Safety Pre-Warning System Based on Data Mining for a Sustainable Food Supply Chain,» Food Control, pp. 73: 223229, 2017.

[120] T. Hu, M. Zheng y L. Zhu, «Research Application of the Internet of Things Monitor Platform in Meat Processing Industry,» de Proceedings of the International Conference on Human-centric Computing and Embedded and Multimedia Computing, 2011.

[121] S. Balachandar y R. Chinnaiyan, «Internet of Things Based Reliable RealTime Disease Monitoring of Poultry Farming Imagery Analytics," de Proceeding of the International Conference on Computer Networks, Big Data and loT, 2020.

[122] Y. Gu, W. Han, L. Zheng y B. Jin, «Using loT Technologies to Resolve the Food Safety Problem - An Analysis Based on Chinese Food Standards.,", de Web Information Systems and Mining, 2012.

[123] A. P. Noletto, S. Loureiro, R. Castro y O. Júnior, «Intelligent Packaging and the Internet of Things in Brazilian Food Supply Chains: The Current State and Challenges,» de Dynamics in Logistics - Proceedings of the 5th International Conference LDIC, Bremen, Springer, 2017, pp. 173-183.

[124] Y. Zhang, B. Chen y X. Lu, «Intelligent Monitoring System on Refrigerator Trucks Based on the Internet of Things,» de Wireless Communications and Applications, 2011.

[125] X. Ai y Y. Zhang, «Modeling Analysis of Intelligent Logistics Distribution Path of Agricultural Products Under Internet of Things Environment,» de Advanced Hybrid Information Processing, 2019.

[126] B. Xu, D. Zhang y W. Yang, «Research on Architecture of the Internet of Things for Grain Monitoring in Storage,» de International Workshop, IoT 2012, Changsha, 2012. 
[127] S. S. Kamble, A. Gunasekaran, H. Parekh y S. Joshi, «Modeling the internet of things adoption barriers in food retail supply chains.,» Journal of Retailing and Consumer Services, pp. 154-168, 2019.

[128] A. Fagerstrøm, N. Eriksson y V. Sigurðsson, «What's the "Thing" in Internet of Things in Grocery Shopping? A Customer Approach,» Procedia Computer Science, 2017.

[129] S. Ulla Shariff, M. G. Gurubasavanna y C. R. Byrareddy, «loT-Based Smart.Food Storage Monitoring and Safety System,» de International Conference on Computer Networks and Communication Technologies, 2018.

[130] N. S. Lakshmi, E. Kavinkartik y E. Prabhu, «loT Based Food Inventory Tracking System,» de Advances in Signal Processing and Intelligent Recognition Systems, 2019.

[131] X. Hong, «Food Ordering Service System Design for Chinese Urban Commuters Based on Internet of Things,» de Distributed, Ambient and Pervasive Interactions, 2017.

[132] S. Jagtap y S. Rahimifard, "The digitisation of food manufacturing to reduce waste - Case study of a ready meal factory,» Waste Management, pp. 387-397, 2019.

[133] Z. Wen, S. Hu, D. De Clercq, M. B. Beck, H. Zhang, H. Zhang, F. Fei y J. Liu, «Design, implementation, and evaluation of an Internet of Things (IoT) network system for restaurant food waste management,» Waste Management, 2018.

[134] J. Chen, Y. Huang, P. Xia, Y. Zhang y Y. Zhong, «Design and implementation of real-time traceability monitoring system for agricultural products supply chain under Internet of Thins architecture,» Concurrency and Computation: Practice and Experience, 2019.

[135] F. Yang, «The Construction of Agricultural Products Traceability System Based on the Internet of Things-The Cases of Pollution-Free Vegetables in Leping of Jiangxi Province,» de Computer and Computing Technologies in Agriculture VII, 2014.

[136] J. Yin, X. Zhang, Q. Lu, C. Xin, C. Liu y Z. Chen, «loT Based Provenance Platform for Vegetables Supplied to Hong Kong,» de Recent Advances in Computer Science and Information Engineering , 2012, pp. 591-596.

[137] K. Furdik, F. Pramudianto, M. Ahlsén, P. Rosengren, P. Kool, S. Zhenyu y A. Schneider, «Food Traceability Chain Supported by the Ebbits loT Middleware,» de Dynamics in Logistics, 2015.

[138] G. Zhao, H. Yu, G. Wang, Y. Sui y L. Zhang, «Applied Research of IOT and RFID Technology in Agricultural Product Traceability System,» de International Conference on Computer and Computing Technologies in Agriculture, 2015.
[139] B. Qu, X. Jing, X. Wang, Y. Li y Y. Liang, «Design on Cucumber Traceability System Based on the Internet of Things,» de Computer and Computing Technologies in Agriculture V, 2012.

[140] L. Liu, P. Liu, W. Ren, Y. Zheng, C. Zhang y J. Wang, «The Traceability Information Management Platform of Duck Product Industry Chain,» de Cloud Computing and Security, 2018.

[141] L. Liu, P. Liu, F. Wen, C. Zhang, R. Zhao, M. Yan y X. Yu, «Information collection system of duck products based on loT,» de EURASIP Journal on Wireless Communications and Networking, 2018.

[142] Z. Li, G. Liu, L. Liu, X. Lai y G. Xu, «loT-based tracking and tracing platform for prepackaged food supply chain,» Industrial Management \& Data Systems, 2017.

[143] R.-Y. Chen, «Autonomous tracing system for backward design in food supply chain,» Food Control, pp. 70-84, 2015

[144] R. Yan, "Optimization approach for increasing revenue of perishable product supply chain with the Internet of Things,» Industrial Management \& Data Systems, 2017.

[145] Z. Pang, Q. Chen, W. Han y L. Zheng, «Value-centric design of the internetof-things solution for food supply chain: Value creation, sensor portfolio and information fusion,» Information Systems Frontiers, pp. 289-319, 2015.

[146] Y. Zhang, L. Zhao y C. Qian, «Modeling of an loT-enabled supply chain for perishable food with two-echelon supply hubs,» Industrial Management \& Data Systems, 2017.

[147] C. Verdouw, J. Wolfert, A. Beulens y A. Rialland, «Virtualization of Food Supply Chains with the Internet of Things,» Journal of Food Engineering, pp. 176: 128-136, 2016

[148] R. Accorsi, M. Bortolini, G. Baruffaldi, F. Pilati y E. Ferrari, «Internet-ofthings Paradigm in Food Supply Chains Control and Management,» Procedia Manufacturing, 2017.

[149] S. Jagtap y S. Rahimifard, «Unlocking the Potential of the Internet of Things to Improve Resource Efficiency in Food Supply Chain,» Innovative Approaches and Applications for Sustainable Rural Development , 2019.

[150] J. Astill, D. Dara, M. Campbell, J. Farber, E. Fraser, S. Sharif y R. Y. Yada, "Transparency in food supply chains: A review of enabling technology solutions,» Trends in Food Science \& Technology, pp. 240-247, 2019.

[151] M. Thibaud, H. Chi, W. Zhoua y S. Piramuthuc, «lnternet of Things (IoT) in high-risk Environment, Health and Safety (EHS) industries: A comprehensive review,» Decision Support Systems, pp. 79-95, 2018.

[152] M. Ben-daya, E. Hassini y Z. Bahroun, «Internet of things and supply chaing management: a literature review,» International Journal of Production Research, pp. 1-24, 2017. 